\title{
THE HODGE GROUP OF AN ABELIAN VARIETY
}

\author{
V. KUMAR MURTY
}

(Communicated by William C. Waterhouse)

\begin{abstract}
Let $A$ be a simple abelian variety of odd dimension, defined over C. If the Hodge classes on $A$ are intersections of divisors, then the semisimple part of the Hodge group of $A$ is as large as it is allowed to be by endomorphisms and polarizations.
\end{abstract}

1. Introduction. Let $A$ be an abelian variety defined over $\mathbf{C}$, and denote by $\mathscr{H}(A)$ its Hodge ring:

$$
\mathscr{H}(A)=\bigoplus_{p}\left(H^{2 p}(A(\mathbf{C}), \mathbf{Q}) \cap H^{p p}\right) .
$$

Denote by $\mathscr{D}(A)$ the subring of $\mathscr{H}(A)$ generated by the elements of $H^{2}(A(\mathbf{C}), \mathbf{Q}) \cap$ $H^{11}$. We denote by $\operatorname{Hod}(A)$ the Hodge group of $A$ introduced by Mumford [3]. It is a connected, reductive algebraic subgroup of $G L(V)$, where $V=H_{1}(A(\mathbf{C}), \mathbf{Q})$. It acts on the cohomology of $A$ and its main property is that

$$
H^{*}(A(\mathbf{C}), \mathbf{Q})^{\operatorname{Hod}(A)}=\mathscr{H}(A) .
$$

We shall also consider a group $L(A)$ which was studied by Ribet [8] and the author [6]. To define it, we fix a polarization $\psi$ of $A$. Then, $L(A)$ is the connected component of the identity of the centralizer of $\operatorname{End}(A) \otimes \mathbf{Q}$ in $S p(V, \psi)$. The definition is independent of the choice of polarization. It is known that $\operatorname{Hod}(A) \subseteq$ $L(A)$ and in [6], it was proved that if $A$ has no simple factor of type III (see $\S 2$ for the definitions), then

$$
\operatorname{Hod}(A)=L(A) \Leftrightarrow \mathscr{H}\left(A^{k}\right)=\mathscr{D}\left(A^{k}\right) \text { for all } k \geq 1 .
$$

Moreover, if $A$ has a factor of type III, then $\mathscr{H}(A) \neq \mathscr{D}(A)$.

Let $G$ be a connected reductive algebraic group defined over $\mathbf{Q}$, and denote by $\mathscr{G}$ its Lie algebra. It is known that there is a unique connected semisimple algebraic subgroup $G_{\text {ss }}$ of $G$ whose Lie algebra is the maximal semisimple subalgebra of $\mathscr{G}$. We refer to $G_{\mathbf{s s}}$ as 'the semisimple part' of $G$.

The purpose of this note is to show that in some cases, the assumption $\mathscr{H}(A)=$ $\mathscr{D}(A)$ is already sufficient to imply that the semisimple parts of $\operatorname{Hod}(A)$ and $L(A)$ are equal. We prove two general results of which the following is a consequence.

Received by the editors July 9, 1986 and, in revised form, August 13, 1987.

1980 Mathematics Subject Classification (1985 Revision). Primary 14C30; Secondary 14K20.

Key words and phrases. Abelian variety, Hodge group, endomorphism algebra.

Research partially supported by NSERC and FCAR grants. 
Proposition. Let $A$ be simple and of odd dimension. Then, $\mathscr{H}(A)=\mathscr{D}(A)$ implies that $\operatorname{Hod}(A)_{\mathrm{ss}}=L(A)_{\mathrm{ss}}$.

A key element in the proof is the classification (cf. Serre [10]) of the simple factors of $\operatorname{Hod}(A)$ and the explicit determination of $L(A)[6]$.

REMARKS. 1. By a classical result of Lefschetz, $\mathscr{D}(A)$ consists of Poincaré duals of algebraic cycles on $A$. Thus, the condition $\mathscr{H}(A)=\mathscr{D}(A)$ implies that the Hodge conjecture holds for $A$. (In fact, it holds in the strong sense that any algebraic cycle on $A$ is algebraically equivalent to an intersection of divisors.)

2. The proof will show that the condition $\mathscr{H}(A)=\mathscr{D}(A)$ can be replaced by the weaker condition that every Hodge cycle on $A$ of codimension $\leq 3$ is contained in $\mathscr{D}(A)$.

3. If we are in a case where $L(A)$ is known to be semisimple, the Proposition shows that $\mathscr{H}(A)=\mathscr{D}(A)$ implies that $\mathscr{H}\left(A^{k}\right)=\mathscr{D}\left(A^{k}\right)$ for all $k \geq 1$. This is because the conditions on $A$ preclude it from being of type III and so $(*)$ can be applied.

4. If $A$ is simple and of $C M$ type, both $\operatorname{Hod}(A)$ and $L(A)$ are tori, and so the Proposition above gives no information. However, Lenstra and Ribet (unpublished) have shown in this case that if $\mathscr{H}(A)=\mathscr{D}(A)$ and $\operatorname{End}(A) \otimes \mathbf{Q}$ is a field which is abelian over $\mathbf{Q}$, then $\operatorname{Hod}(A)=L(A)$. It is an interesting problem to study how small $\operatorname{Hod}(A)$ can be, relative to $L(A)$, when $A$ is of $C M$ type. Liem Mai [12] has recently obtained lower bounds for the dimension of $\operatorname{Hod}(A)$, in some cases, and Dodson [1] has shown that this dimension must satisfy some congruence conditions.

5. Tanke'ev has shown that if $A$ is simple and of prime dimension, $\operatorname{Hod}(A)=$ $L(A)$ and $\mathscr{H}\left(A^{k}\right)=\mathscr{D}\left(A^{k}\right)$ for all $k \geq 1$. The proof has been simplified by Ribet [8] who also showed that the conclusion is valid for a slightly larger class of abelian varieties.

I am grateful to Kenneth Ribet for helpful comments on an earlier version of this note.

2. Lemmas. Fix an algebraic closure $\overline{\mathbf{Q}}$ of $\mathbf{Q}$ and an embedding $\overline{\mathbf{Q}} \hookrightarrow \mathbf{C}$. Let $\rho$ denote complex conjugation. We write

$$
\Delta(A)=\operatorname{End}(A) \otimes_{Z} \mathbf{Q}
$$

and let $E$ be a maximal commutative semisimple subalgebra of $\Delta(A)$. Thus, $E$ is a product of fields $E=E_{1} \times \cdots \times E_{r}$ and by the classification of Albert (cf. Mumford $[4, \S 21])$ each factor is either a $C M$ field or a totally real field. We say that $A$ is of type III if $\Delta(A)$ is a totally definite quaternion division algebra over a totally real field, and $A$ is of type IV if $\Delta(A)$ is a division algebra over a $C M$ field.

We have a decomposition $V \otimes \mathbf{C}=\prod V_{\sigma}$ indexed by the set $\Sigma$ of homomorphisms $\sigma: E \otimes \mathbf{C} \rightarrow \mathbf{C}$, where

$$
V_{\sigma}=(V \otimes \mathbf{C}) \otimes_{E \otimes \mathbf{C}, \sigma} \mathbf{C} .
$$

Let $H=\operatorname{Hod}(A)_{/ \mathrm{C}}$ and $L=L(A)_{/ \mathrm{C}}$. Each $V_{\sigma}$ is an $L$ module and hence, also an $H$ module. Denote by $L_{\sigma}$ the projection of $L$ to $G L\left(V_{\sigma}\right)$. 
LEMMA 1. Each $V_{\sigma}$ is a simple $H$ module, and the following are equivalent:

(i) $V_{\sigma_{1}} \sim V_{\sigma_{2}}$ as $H$ modules,

(ii) $V_{\sigma_{1}} \sim V_{\sigma_{2}}$ as $L$ modules.

PROOF. It follows easily from the definitions that

$$
\operatorname{End}_{\text {Hod }(A)}(V)=\operatorname{End}_{L(A)} V=\Delta(A) .
$$

As $E$ is a maximal commutative semisimple subalgebra, we have

$$
\operatorname{End}_{\text {Hod }(A), E} V=E \text {. }
$$

Complexifying, we see that for any $\sigma \in \Sigma$, we have

$$
\operatorname{End}_{H} V_{\sigma}=\mathbf{C} \text {. }
$$

This proves the first assertion. To see the equivalence of (i) and (ii), note that for any $\sigma_{1}, \sigma_{2} \in \Sigma$, we have $\operatorname{Hom}_{L}\left(V_{\sigma_{1}}, V_{\sigma_{2}}\right) \hookrightarrow \operatorname{Hom}_{H}\left(V_{\sigma_{1}}, V_{\sigma_{2}}\right)$. Now

$$
\prod \operatorname{Hom}_{H}\left(V_{\sigma_{1}}, V_{\sigma_{2}}\right)=\prod \operatorname{Hom}_{L}\left(V_{\sigma_{1}}, V_{\sigma_{2}}\right)
$$

as both sides are equal to $\Delta(A) \otimes \mathbf{C}$. (The product on both sides is over all pairs $\sigma_{1}, \sigma_{2} \in \Sigma$.) The result follows.

Denote by $E_{i}^{+}$the maximal totally real subfield of $E_{i}(1 \leq i \leq r)$ and set $E^{+}=E_{1}^{+} \times \cdots \times E_{r}^{+}$. For each homomorphism $\lambda: E^{+} \otimes \mathbf{R} \rightarrow \mathbf{R}$, set

$$
X_{\lambda}=(V \otimes \mathbf{R}) \otimes_{E^{+} \otimes \mathbf{R}, \lambda} \mathbf{R} .
$$

Since $L(A)$ commutes with $E$, it acts on $X_{\lambda}$. We write $L_{\lambda}$ for the projection $L(A) \rightarrow G L\left(X_{\lambda}\right)$.

Suppose that every simple factor of $A$ is of type IV. Then, for every $\lambda, X_{\lambda}$ has a natural complex structure and the polarization induces a Hermitian form $\omega_{\lambda}$ on $X_{\lambda}$. Denote by $U\left(X_{\lambda}, \omega_{\lambda}\right)$ the unitary group with respect to $\omega_{\lambda}$.

LEMMA 2. If every simple factor of $A$ is of type IV, then for every $\lambda, L_{\lambda}=$ $U\left(X_{\lambda}, \omega_{\lambda}\right)$.

PROOF. This follows from Lemma 2.1 and Lemma 2.3 of [6].

We know that

$$
H \subseteq L \subseteq \prod G L\left(V_{\sigma}\right)
$$

Let $S=H_{\mathrm{ss}}$ and $R=L_{\mathrm{ss}}$. Let $H_{\sigma}$ denote the projection of $H$ to $G L\left(V_{\sigma}\right)$, and $S_{\sigma}$ the projection of $S$ to $S L\left(V_{\sigma}\right)$.

LEMMA 3. If $V_{\sigma}$ is of odd dimension (over $\mathbf{C}$ ), then every simple component of $S_{\sigma}$ is of type $\mathbf{A}$. Moreover, if $V_{\sigma}=V_{1, \sigma} \otimes V_{2, \sigma} \otimes \cdots \otimes V_{m, \sigma}$ is a decomposition of $V_{\sigma}$ induced by the decomposition of $S_{\sigma}$ into simple components, each $V_{i, \sigma}$ is an exterior power of the standard representation (i.e. a fundamental representation).

This is [10, Corollary to Proposition 8].

LEMMA 4. Let $S_{i}(1 \leq i \leq r)$ be complex simple Lie groups of type $\mathbf{A}_{m(i)}$, $m(i) \geq 3$ for all $i$. Let $Y_{1}, \ldots, Y_{r}$ be fundamental representations of $S_{1}, \ldots, S_{r}$ (respectively). Let

$$
S=S_{1} \times S_{2} \times \cdots \times S_{r} \quad \text { and } \quad Y=Y_{1} \otimes Y_{2} \otimes \cdots \otimes Y_{r} .
$$


If the $S$ modules $\bigwedge^{2} Y$ and $\bigwedge^{3} Y$ are irreducible, then $r=1$ and $Y$ is the standard representation of $S$ or its dual.

PROOF. For each $i$,

$$
\operatorname{Sym}^{2}\left(Y_{1}\right) \otimes \cdots \otimes \operatorname{Sym}^{2}\left(Y_{i-1}\right) \otimes \bigwedge^{2} Y_{i} \otimes \operatorname{Sym}^{2}\left(Y_{i+1}\right) \otimes \cdots \otimes \operatorname{Sym}^{2}\left(Y_{r}\right)
$$

is an $S$ submodule of $\bigwedge^{2} Y$. Thus, $\bigwedge^{2} Y$ is reducible unless $r=1$. Now, let $W$ denote the standard representation of $S$ and set $w=\operatorname{dim} W$. Suppose that $Y=\wedge^{j} W$. We may assume that $j \leq w / 2$ by replacing $Y$ with its dual if necessary. In the notation of Jacobson [2, Chapters 4 and 8], the highest weight of the exterior square of $Y$ is $\alpha_{j}+\alpha_{j+1}$. By the Weyl dimension formula, we must then have

$$
\left(\begin{array}{c}
\left(\begin{array}{c}
w \\
j
\end{array}\right. \\
2
\end{array}\right)=\operatorname{dim} \bigwedge^{2} Y=\frac{3(w+1)}{(j+1)(w-j+2)}\left(\begin{array}{c}
w \\
j-1
\end{array}\right)\left(\begin{array}{c}
w \\
j+1
\end{array}\right) .
$$

Easy estimates show that this is possible only if $j \leq 2$. If $j=2$, the highest weight of the exterior cube of $Y$ is $2 \alpha_{1}+\alpha_{4}$ and again, by the dimension formula, we must have

$$
\left(\begin{array}{c}
\left(\begin{array}{c}
w \\
2 \\
3
\end{array}\right)
\end{array}\right)=\operatorname{dim} \bigwedge^{3} Y=\frac{1}{3}(w+1)(w+2)\left(\begin{array}{c}
w \\
4
\end{array}\right)
$$

which forces $w \leq 2$. Thus, we must have $j=1$ and the lemma is proved.

LEMMA 5. Let $W_{1}, W_{2}$ be two finite dimensional complex vector spaces. Let $\mathscr{S}_{1}, \mathscr{S}_{2}$ be simple complex Lie subalgebras of $\mathscr{L} \mathscr{L}\left(W_{1}\right), \mathscr{G} \mathscr{L}\left(W_{2}\right)$ (respectively) of type A, B or C. Let $\mathscr{S}$ be a Lie subalgebra of $\mathscr{S}_{1} \times \mathscr{S}_{2}$ whose projection to each factor is surjective. Then, either $\mathscr{S}=\mathscr{S}_{1} \times \mathscr{S}_{2}$ or $\mathscr{S}$ is the graph of an isomorphism $\mathscr{S}_{1} \simeq \mathscr{S}_{2}$ induced by an isomorphism $W_{2} \simeq W_{1}$ or $\check{W}_{1}$ of $\mathscr{S}_{\text {-modules. }}$

ProOF. For $i=1,2$, let $\pi_{i}: \mathscr{S} \rightarrow \mathscr{S}_{i}$ denote the $i$ th projection and set $\mathscr{N}_{i}=$ $\operatorname{ker} \pi_{3-i}$. We may view $\mathscr{N}_{i}$ as an ideal of $\mathscr{S}_{i}$ and hence, $\mathscr{N}_{i}=0$. By Goursat's Lemma (see Ribet $[7, \S 5]$ ), it follows that $\mathscr{S}=\mathscr{S}_{1} \times \mathscr{S}_{2}$ or $\mathscr{S}$ is the graph of an isomorphism $\mathscr{S}_{1} \simeq \mathscr{S}_{2}$. In the latter case, it follows that $\mathscr{S}_{1}, \mathscr{S}_{2}$ are both of the same type. If they are of type $\mathbf{B}$ or $\mathbf{C}$, it follows that $W_{1} \simeq W_{2}$ as $\mathscr{S}$-modules, since every automorphism of such algebras is inner [2, p. 281]. If they are of type A, then $W_{1} \simeq W_{2}$ or $\check{W}_{2}$ as $\mathscr{S}$ modules, since, modulo inner automorphisms, the only automorphism of such an algebra is $g \mapsto-{ }^{t} g$.

LEMMA 6. Let I be a finite set and for each $\sigma \in I$, let $\mathscr{S}_{\sigma}$ be a finite dimensional complex simple Lie algebra. Let $\mathscr{A}, \mathscr{B}$ be two algebras such that

(a) $\mathscr{A} \subseteq \mathscr{B}$.

(b) $\mathscr{B}$ is a subalgebra of $\prod \mathscr{S}_{\sigma}$ and the projection to each $\mathscr{S}_{\sigma}$ is surjective.

(c) $\mathscr{A}, \mathscr{B}$ have equal images on $\mathscr{S}_{\sigma} \times \mathscr{S}_{\tau}$ for all pairs $(\sigma, \tau) \in I \times I, \sigma \neq \tau$. Then, $\mathscr{A}=\mathscr{B}=\prod \mathscr{S}_{\sigma}$ where the $\sigma$ range over a certain subset of $I$.

This is [9, Lemma 4.6].

3. Main results. We retain the notation of the previous section. Thus, $A$ is an abelian variety, $V=H_{1}(A(\mathbf{C}), \mathbf{Q})$ and $E$ is a maximal commutative semisimple subalgebra of $\Delta(A)$. 
THEOREM 1. Suppose that $E$ is a product of $C M$ fields and that $V$ is free over $E$ of odd rank $m$. Suppose that $\mathscr{H}(A)=\mathscr{D}(A)$. Then $\operatorname{Hod}(A)_{\mathrm{ss}}=L(A)_{\mathrm{ss}}$.

PROOF. First, we check that every simple factor of $A$ is of type IV. We have $E=E_{1} \times \cdots \times E_{r}$, with $E_{i}$ a $C M$ field. There is a corresponding decomposition up to isogeny $A \sim A_{1} \times \cdots \times A_{r}$. As $E_{i}$ is a field, $A_{i}$ is a power of a simple abelian variety, say $A_{i}=B_{i}^{n(i)}$. To show that $B_{i}$ is of type IV, it suffices to show that $\operatorname{End}\left(B_{i}\right) \otimes \mathbf{Q}$ is not a quaternion division algebra $D_{i}$ over a totally real field $F_{i}$. Suppose that it is and let $x_{i}=\operatorname{dim}_{D_{i}} H_{1}\left(B_{i}(\mathbf{C}), \mathbf{Q}\right)$. As $E_{i}$ is a maximal commutative subfield of

$$
\Delta\left(A_{i}\right)=M_{n(i)}\left(D_{i}\right)
$$

we have

$$
m=\frac{\operatorname{dim} V_{i}}{\left[E_{i}: \mathbf{Q}\right]}=\frac{n(i) x_{i}\left[D_{i}: \mathbf{Q}\right]}{2 n(i)\left[F_{i}: \mathbf{Q}\right]}=2 x_{i}
$$

This contradicts our assumption that $m$ is odd. Thus, every simple factor of $A$ is of type IV.

Now, for each homomorphism $\lambda: E^{+} \otimes \mathbf{R} \rightarrow \mathbf{R}$, we have

$$
X_{\lambda} \otimes \mathbf{C}=V_{\sigma} \oplus V_{\rho \sigma}
$$

where $\sigma$ is an extension of $\lambda$ to a map $E \otimes \mathbf{C} \rightarrow \mathbf{C}$. Lemma 2 implies that as $L$ modules,

$$
V_{\rho \sigma} \simeq \check{V}_{\sigma} .
$$

Moreover, Lemma 1 implies that the $V_{\sigma}$ are all simple as $H$ modules. Let $R_{\sigma}$ denote the image of $R$ in $S L\left(V_{\sigma}\right)$. By Lemma 2, $R_{\sigma}=S L\left(V_{\sigma}\right)$. As $\operatorname{dim}_{\mathbf{C}}\left(V_{\sigma}\right)=m$ is odd, $m=1$ or $m \geq 3$. In the first case, $S_{\sigma}=R_{\sigma}=1$. We may thus suppose that $m \geq 3$. By Lemma 3, there is an isogeny $S_{\sigma} \sim S_{1, \sigma} \times \cdots \times S_{r, \sigma}$ with each $S_{i, \sigma}$ a simple group of type $\mathbf{A}$. In the corresponding decomposition $V_{\sigma}=V_{1, \sigma} \otimes \cdots \otimes V_{r, \sigma}$, each $V_{i, \sigma}$ is a fundamental representation of $S_{i, \sigma}$.

By [6, Lemmas 2.1, 3.4 and 3.6] and our assumption that $\mathscr{H}(A)=\mathscr{D}(A)$, we see that

$$
H^{*}(A(\mathbf{C}), \mathbf{Q})^{\operatorname{Hod}(A)}=\mathscr{H}(A)=\mathscr{D}(A)=H^{*}(A(\mathbf{C}), \mathbf{Q})^{L(A)} .
$$

Thus, for any sequence $\{i(\sigma)\}_{\sigma}$ of positive integers,

$$
\text { the } H \text { and } L \text { invariants of } \bigotimes_{\sigma} \bigwedge^{i(\sigma)} \check{V}_{\sigma} \text { must agree. }
$$

In particular, utilising (1), we see that for any $\sigma$, and any integer $i$ with $1 \leq i \leq m$, we must have

$$
\operatorname{dim} \operatorname{End}_{H_{\sigma}}\left(\bigwedge^{i} V_{\sigma}\right)=\operatorname{dim} \operatorname{End}_{L_{\sigma}}\left(\bigwedge^{i} V_{\sigma}\right)=1
$$

Since $H_{\sigma} \subseteq L_{\sigma}=G L\left(V_{\sigma}\right)$, the center $Z_{\sigma}$ of $H_{\sigma}$ consists at most of scalars. Thus (2) is equivalent to

$$
\operatorname{End}_{S_{\sigma}}\left(\bigwedge^{i} V_{\sigma}\right)=\mathbf{C}
$$


for all $\sigma$. In particular, $\bigwedge^{2} V_{\sigma}$ and $\bigwedge^{3} V_{\sigma}$ are irreducible as $S_{\sigma}$ modules. Since $S_{\sigma} \subseteq R_{\sigma}$ and $R_{\sigma}$ acts on $V_{\sigma}$ by the standard representation, Lemma 4 implies that

$$
S_{\sigma}=S L\left(V_{\sigma}\right)=R_{\sigma} .
$$

In particular, the Lie algebra $\mathscr{S}_{\sigma}$ of $S_{\sigma}$ is $\mathscr{S} \mathscr{L}\left(V_{\sigma}\right)$. Now, for $\sigma, \tau \in \Sigma, \sigma \neq \tau$, let $S_{\sigma, \tau}$ (respectively, $R_{\sigma, \tau}$ ) denote the image of $S$ (respectively, $R$ ) in $S L\left(V_{\sigma}\right) \times S L\left(V_{\tau}\right)$. Let $\mathscr{S}_{\sigma, \tau}$ (respectively, $\mathscr{R}_{\sigma, \tau}$ ) denote the Lie algebra of $S_{\sigma, \tau}$ (respectively, $R_{\sigma, \tau}$ ). Goursat's Lemma implies that

$$
\mathscr{S}_{\sigma, \tau}=\mathscr{S} \mathscr{L}\left(V_{\sigma}\right) \times \mathscr{S} \mathscr{L}\left(V_{\tau}\right)
$$

or $\mathscr{S}_{\sigma, \tau}$ is the graph of an isomorphism

$$
\mathscr{S} \mathscr{L}\left(V_{\sigma}\right) \simeq \mathscr{S} \mathscr{L}\left(V_{\tau}\right)
$$

If (4) occurs, then we see that

$$
\mathscr{R}_{\sigma, \tau}=\mathscr{S} \mathscr{L}\left(V_{\sigma}\right) \times \mathscr{S} \mathscr{L}\left(V_{\tau}\right)
$$

also, since $\mathscr{S}_{\sigma, \tau} \subseteq \mathscr{R}_{\sigma, \tau}$.

Now suppose (5) occurs. Lemma 5 and (1) imply that

$$
V_{\sigma} \simeq V_{\tau} \text { or } V_{\rho \tau}
$$

as $S$ modules. If $\tau=\rho \sigma$, then it is clear that $\mathscr{R}_{\sigma, \tau}$ is also the graph of the isomorphism (5). We may therefore suppose that $\tau$ is different from $\rho \sigma$. Consider the $H$ submodule of $H_{4}(A(\mathbf{C}), \mathbf{C})$ given by

$$
W=V_{\sigma} \otimes V_{\rho \sigma} \otimes V_{\tau} \otimes V_{\rho \tau} .
$$

From (6), it follows that as $S$ modules,

$$
\begin{aligned}
W & \simeq V_{\sigma} \otimes V_{\sigma} \otimes V_{\rho \sigma} \otimes V_{\rho \sigma} \simeq \operatorname{End}\left(V_{\sigma} \otimes V_{\sigma}\right) \\
& \simeq \operatorname{End}\left(\bigwedge^{2} V_{\sigma} \oplus \operatorname{Sym}^{2} V_{\sigma}\right) .
\end{aligned}
$$

The latter contains a two dimensional subspace

$$
W_{\sigma, \tau}=\operatorname{End}_{S}\left(\bigwedge^{2} V_{\sigma}\right) \oplus \operatorname{End}_{S}\left(\operatorname{Sym}^{2} V_{\sigma}\right)
$$

on which $S$ acts trivially. The center of $H$ acts trivially on $W$, and hence also on $W_{\sigma, \tau}$. It follows that $H$ acts trivially on $W_{\sigma, \tau}$. By our assumption (**), $L$ also acts trivially on this space. It follows that (6) holds as $L$ modules also, for otherwise, the subspace of $W$ on which $L$ acts trivially is only one dimensional. Hence, we deduce that $\mathscr{R}_{\sigma, \tau}$ is the graph of an isomorphism $\mathscr{S} \mathscr{L}\left(V_{\sigma}\right) \simeq \mathscr{S} \mathscr{L}\left(V_{\tau}\right)$ and in particular, $\mathscr{R}_{\sigma, \tau}=\mathscr{S}_{\sigma, \tau}$.

Now, appealing to Lemma 6, we conclude that

$$
\mathscr{S}=\mathscr{R}=\prod \mathscr{S} \mathscr{U}\left(X_{\lambda}\right)
$$

where $\lambda$ ranges over a certain subset of the homomorphisms $E^{+} \otimes \mathbf{R} \rightarrow \mathbf{R}$. Since $S$ and $R$ are connected, and $S \subseteq R$, it follows that $S=R$. This proves the Theorem.

REMARK. In fact, it can be shown that $H_{\sigma}=G L\left(V_{\sigma}\right)$ and that the dimension of the center of $H$ is at least 2. Hence, if $\operatorname{dim}_{\mathbf{Q}} E \leq 4$, then $\operatorname{Hod}(A)=L(A)$.

The next theorem is a slight generalization of a result of Tanke'ev [11] who proved it in the case $\Delta(A)=E$. The case $m=1$ is a special case of $[5$, Theorem 4.1]. 
THEOREM 2. Suppose that $E$ is a product of totally real fields and that $V$ is free over $E$ of rank $2 m, m$ odd. Then $\operatorname{Hod}(A)=L(A)$. In particular, $\mathscr{H}\left(A^{k}\right)=\mathscr{D}\left(A^{k}\right)$ for all $k \geq 1$.

ProOF. As before, for each homomorphism $\sigma: E \otimes \mathbf{C} \rightarrow \mathbf{C}$, we have the $H$ module $V_{\sigma}$ which is a $2 m$ dimensional $\mathbf{C}$ vector space. Write $H_{\sigma} \sim H_{1, \sigma} \times \cdots \times H_{r, \sigma}$ where each $H_{i, \sigma}$ is a simple group. Write $V_{\sigma}=V_{1, \sigma} \otimes \cdots \otimes V_{r, \sigma}$ for the corresponding decomposition of $V_{\sigma}$. By Lemma 1 , each $V_{\sigma}$ is a simple $H_{\sigma}$ module. As $E$ is a product of totally real fields, the polarization $\psi$ induces an alternating form $\psi_{\sigma}$ on $V_{\sigma}$ and $H_{\sigma} \subseteq L_{\sigma} \subseteq S p\left(V_{\sigma}, \psi_{\sigma}\right)$. (Here, $L_{\sigma}$ is the projection of $L$ to $G L\left(V_{\sigma}\right)$.) Hence, each $V_{i, \sigma}$ is either a symplectic or orthogonal representation of $H_{i, \sigma}$. Since a symplectic representation has even dimension, exactly one of the $V_{i, \sigma}$ is symplectic and the others must be orthogonal. From [10, Proposition 7 and the Appendix], we see that when $m$ is odd, there are the following possibilities:

(i) $H_{i, \sigma}$ is of type $\mathbf{A}_{n}(n \geq 1)$ and $V_{i, \sigma}$ is an exterior power of the standard representation. Here, $\operatorname{dim} V_{i, \sigma}=\left(\begin{array}{c}n+1 \\ k\end{array}\right)$ for some $k \leq n$.

(ii) $H_{i, \sigma}$ is of type $\mathbf{C}_{n}(n \geq 2)$ and $V_{i, \sigma}$ is the standard representation. Here, $\operatorname{dim} V_{i, \sigma}=2 n$ and $V_{i, \sigma}$ is symplectic.

(iii) $H_{i, \sigma}$ is of type $\mathbf{D}_{n}(n \geq 4)$ and $V_{i, \sigma}$ is the standard representation. Here, $\operatorname{dim} V_{i, \sigma}=2 n$ and $V_{i, \sigma}$ is orthogonal.

Suppose $H_{i, \sigma}$ is of type $\mathbf{A}_{n}$. For $V_{i, \sigma}$ to be symplectic or orthogonal, we must have $n$ odd, say $n+1=2 n_{0}$, and $k=n_{0}$. Moreover, $V_{i, \sigma}$ is symplectic when $n_{0}$ is odd and orthogonal when $n_{0}$ is even. Now,

$$
\operatorname{dim} V_{i, \sigma}=\left(\begin{array}{c}
n+1 \\
k
\end{array}\right)=\left(\begin{array}{c}
2 n_{0} \\
n_{0}
\end{array}\right)
$$

is always even and is divisible by 4 if $n_{0}$ is odd. Thus, $m$ odd implies that $V_{i, \sigma}$ cannot be symplectic. But in this case, some other $V_{j, \sigma}$ is symplectic and this would give $4 \mid 2 m$ and so, case (i) cannot occur. But, as $\operatorname{dim} V_{i, \sigma}$ is even in both the remaining cases, we must have $r=1$ and $H_{\sigma}$ of type $\mathbf{C}_{n}$, i.e. $H_{\sigma}=S p\left(V_{\sigma}, \psi_{\sigma}\right)=L_{\sigma}$. Again, by taking Lie algebras and appealing to Lemmas 1,5 and 6 , it follows that

$$
H=L=\prod S p\left(V_{\sigma}, \psi_{\sigma}\right) .
$$

Finally, we check that no simple factor of $A$ is of type III by a dimension calculation as in the proof of Theorem 1. Now by [6, Theorem 3.1] (stated as (*) in the Introduction), it follows that $\mathscr{H}\left(A^{k}\right)=\mathscr{D}\left(A^{k}\right)$ for all $k \geq 1$. This proves the theorem.

Finally, we combine the two theorems to prove the result stated in the Introduction.

Proof of the Proposition. As $A$ is simple, any maximal commutative semisimple subalgebra of $\Delta(A)$ must in fact be a field $E$, (say). Moreover, $E$ is totally real or a $C M$ field. In the first case, $E$ acts on $\operatorname{Lie}(A)$ and so $[E: \mathbf{Q}]$ divides $\operatorname{dim} A$. Thus, $\operatorname{dim}_{E} V=2 m, m$ odd. The Proposition follows in this case from Theorem 2. In the second case, $[E: \mathbf{Q}]$ is even and so $\operatorname{dim}_{E} V=m$ is odd. The Proposition follows in this case from Theorem 1. 


\section{REFERENCES}

1. B. Dodson, On the Mumford-Tate group of an abelian variety with complex multiplication, J. Algebra 111 (1987), 49-73.

2. N. Jacobson, Lie algebras, Interscience, 1962.

3. D. Mumford, Families of abelian varieties, Algebraic Groups and Discontinuous Subgroups (A. Borel and G. Mostow, eds.), Proc. Sympos. Pure Math., vol. 9, Amer. Math. Soc., Providence, R. I., 1966, pp. 347-351.

4. __ Abelian varieties, Tata Institute Lecture Notes, Oxford, 1974.

5. V. Kumar Murty, Algebraic cycles on abelian varieties, Duke Math. J. 50 (1983), 487-504.

6. __ Exceptional Hodge classes on certain abelian varieties, Math. Ann. 268 (1984), 197-206.

7. K. Ribet, Galois action on division points of abelian varieties with real multiplication, Amer. J. Math. 98 (1976), 751-804.

8. __ Hodge classes on certain types of abelian varieties, Amer. J. Math. 105 (1983), 523-538.

9. __ Twists of modular forms and endomorphisms of abelian varieties, Math. Ann. 253 (1980), 43-62.

10. J.-P. Serre, Groupes algébriques associés aux modules de Hodge-Tate, Astérisque 65 (1979), 155-188.

11. S. G. Tanke'ev, On algebraic cycles on surfaces and abelian varieties, Math. USSR Izv. 18 (1982), 349-380.

12. Liem Mai, Lower bounds for the rank of a CM type, M.Sc. thesis, Concordia University, 1987.

Department of Mathematics, Concordia University, Montreal, Canada

Current address: Department of Mathematics, University of Toronto, Toronto, Canada M5S 1A1 